\title{
Towards a finite- $N$ hologram
}

\section{Chethan Krishnan and K.V. Pavan Kumar}

Center for High Energy Physics, Indian Institute of Science, Bangalore 560012, India

E-mail: chethan.krishnan@gmail.com, kumar pavan56@gmail.com

ABSTRACT: We suggest that holographic tensor models related to SYK are viable candidates for exactly (ie., non-perturbatively in $N$ ) solvable holographic theories. The reason is that in these theories, the Hilbert space is a spinor representation, and the Hamiltonian (at least in some classes) can be arranged to commute with the Clifford level. This makes the theory solvable level by level. We demonstrate this for the specific case of the uncolored $O(n)^{3}$ tensor model with arbitrary even $n$, and reduce the question of determining the spectrum and eigenstates to an algebraic equation relating Young tableaux. Solving this reduced problem is conceptually trivial and amounts to matching the representations on either side, as we demonstrate explicitly at low levels. At high levels, representations become bigger, but should still be tractable. None of our arguments require any supersymmetry.

Keywords: 1/N Expansion, AdS-CFT Correspondence, Black Holes in String Theory

ARXIV EPRINT: 1706.05364 


\section{Contents}

1 Introduction 1

2 The setup 3

2.1 Holographic tensor model 3

2.2 Making Clifford work 3

2.3 Level 0 and level 1

2.4 Level 2 and higher 5

$\begin{array}{lll}3 & \text { Diagonalization via Young tableaux } & 6\end{array}$

$\begin{array}{lll}3.1 \text { Comments } & 7\end{array}$

4 Conclusion $\quad 8$

$\begin{array}{lll}\text { A Explicit diagonalization of level } 2 & 9\end{array}$

$\begin{array}{ll}\text { B Counting at level 2 } & 10\end{array}$

\section{Introduction}

Holography has provided us with our most complete definition of quantum gravity so far. This means that we know perfectly well-defined quantum mechanical systems which contain classical gravity in some appropriate limit. See $[1,2]$ for some discussions on the general philosophy of holography. However, having a definition of quantum gravity is not the same as being able to extract the quantum physics of gravity from it. For that, we need to not just know what the theory is, we also need to usefully solve it at non-zero values of Planck's constant. This, has largely remained a challenge.

Typically in holographic theories, we have a paramter $N$ which can loosely be thought of as the inverse of a (dimensionless) Planck's constant. The usual situation is that we have reasonable control on the theory in the classical $N \rightarrow \infty$ limit, and perhaps also perturbatively to low orders [3-5] in $1 / N$. In some cases [6-11] we even know the perturbative quantum corrections to all orders in $1 / N$. This means that we know at least some models were holographic theories are under reasonable perturbative control. To the best of our knowledge however, there is no known example where a holographic theory is fully solvable exactly for arbitrary tunable values of $N$. This is not just a matter of being pedantic, there are strong reasons [12-18] to think that unitarization of black holes (for example), will require ${ }^{1}$ one to understand non-perturbative corrections in $1 / N$.

\footnotetext{
${ }^{1}$ Let us emphasize a few things here, however: (1) The double-scaled matrix models of [6-11] are dual to topological strings and do not contain black holes, so this criticism is not directly relevant to them. (2)
} 
In this paper, we will argue that the holographic tensor models that have recently emerged [26-35] in the context of SYK models [36-75] are a potential candidate for such fully solvable theories. See [76-80] for other related works on tensor models.

Exact diagonalization of the holographic tensor models might seem like a hopeless task at first. Indeed, in [30, 31] these theories were numerically diagonalized for small (and fixed) values of some appropriately chosen $N$. But it was also observed that as the $N$ was increased, the matrix sizes grew exponentially as $\sim \exp \left(N^{\#}\right)$ where $\#$ is $\geq 1$, and depends on the specific model. This makes exact diagonalization essentially impossible fairly quickly even with modern computers. Also, a numerical diagonalization is not as useful for many crucial purposes from a holographic point of view. In particular, constructing singlets in order to make contact with a "closed string" bulk perspective is much more intuitive when one has at least some analytic understanding. Furthermore, even in the simplest cases considered in $[30,31]$ explicit diagonalization on a computer was too demanding for the eigenvectors to be found: only the eigenvalues were found in [30, 31].

Nontheless, in this paper we will argue that determining the eigenvalues and eigenvectors of Hamiltonians of (at least some classes of) holographic tensor models can be reduced to highly tractable combinatorical questions regarding representation decompositions. We will support this claim by simplifying the diagonalization problem for the even rank uncolored $O(n)^{3}$ tensor models using a basic observation: that the Hilbert space of these theories forms a spinor representation, and that by appropriate choice of the spinor raising and lowering operators, one can ensure that the Hamiltonian commutes with the Clifford level. The advantage of this is that it enables us to diagoinalize the Hamiltonian level by level, which in turn reduces it to a problem of breaking down tensor products of representations in the language of Young tableaux. We believe that a complete solution of this latter problem is possible, but it becomes cumbersome and requires a systematic approach when it comes to the representations at high levels, so we will present it in a forthcoming publication [85]. In this paper, we will derive the statement of the reduced problem in terms of Young tableaux (equation (3.1) is our main result), as well as present its solution for Clifford levels 0,1 and 2 (which automatically yields the solution for the highest, penultimate and pre-penultimate Clifford levels as well). The diagonalization problem reduces to the algebraic question of equating the representation content on either side of this

It is not necessary that we be able to solve the theory completely at finite $N$ before we can answer any non-pertubative question about quantum gravity. For example, localization [19] has been useul in exactly evaluating some partition functions and operators non-perturbatively in contexts where supersymmetry is available. In this and other cases, some quantities are computable exactly. But what we mean by full solvability in this paper is that (in principle) we know all the correlation functions in the theory, or equivalently, that we know the spectrum and the eigenstates. (3) To answer detailed dynamical questions, especially regarding local bulk physics (like in the case of black hole unitarity etc.), it seems that a far more detailed understanding of non-perturbative questions than what we currently have access to, will be necessary. (4) The theories we discuss in this paper, and claim are exactly solvable, are expected to be dual to asymptotically $\mathrm{AdS}_{2}$ spaces which have substantially limited dynamics [20] than full higher dimensional gravity. So these theories are likely not general enough to fully answer questions related to black hole unitarity. But they are certainly dynamically more rich than the (quasi-)topological cases discussed in [611]. They also exhibit saturated chaos [21, 22] as expected in black hole physics [23], which is not the case in the matrix model [24], see also [25]. 
equation. Solving it is conceptually trivial, as our low level calculations will demonstrate. But at high levels will require working with big representations - perhaps on a computer.

In the next two sections, we will present the set up and our results. In a final section, we make various comments on related questions, implications of our results and future directions.

\section{The setup}

\subsection{Holographic tensor model}

The theory we consider in this paper is an uncolored tensor model, written down in [27] adapting the colored model in [26]. In particular, the Hamiltonian that we wish to diagonalize is:

$$
H=\psi^{i j k} \psi^{i l m} \psi^{p j m} \psi^{p l k}
$$

where each of the tensor indices take values from 1 to $n$. The theory is quantum mechanical, ie., there is only a time diection and no space. Note that contractions between different tensors can occur only between the same slots on each tensor. The components of the tensors are "Majorana fernions", by which condensed matter theorists mean real objects that satisfy Clifford algebra, aka Gamma matrices. We have set the coupling to unity for conveneince, it can always be reinstated by dimensional analysis. This Hamiltonian, by construction, is $O(n)^{3}$ invariant. The fermionic tensors obey the following commutation relations:

$$
\left\{\psi^{i_{1} j_{1} k_{1}}, \psi^{i_{2} j_{2} k_{2}}\right\}=\delta^{i_{1} i_{2}} \delta^{j_{1} j_{2}} \delta^{k_{1} k_{2}}
$$

In the rest of the paper, we work with the case of even $n$. We believe that an adaptation of the technology we develop here should also exist for the odd $n$ case. But in this paper our goal is to demonstrate a proof of principle, so we will be minimalistic.

\subsection{Making Clifford work}

For even $n$, we can define the following quantities:

$$
\Gamma^{i j k^{ \pm}} \equiv \psi^{i j k^{ \pm}}=\frac{1}{\sqrt{2}}\left(\psi^{i j k} \pm i \psi^{i j(k+1)}\right)
$$

where $k^{ \pm}$take values from 1 to $\frac{n}{2}$. These redefined tensors has the following anticommutation relations:

$$
\left\{\psi^{i j k^{+}}, \psi^{l m n^{+}}\right\}=0 ; \quad\left\{\psi^{i j k^{-}}, \psi^{l m n^{-}}\right\}=0 ; \quad\left\{\psi^{i j k^{+}}, \psi^{l m n^{-}}\right\}=\delta^{i l} \delta^{j m} \delta^{k^{+}, n^{-}}
$$

Since the Clifford algebra (2.2) is realized in the Hilbert space of the theory, it is natural to try and rewrite the Hamiltonian in a form that takes advantage of it. Note that the algebra (2.2) is not the Clifford algebra of $\mathrm{SO}(n)$, but of $\mathrm{SO}\left(n^{3}\right)$, so one might think that working with Clifford raising and lowering operators that transform in $\mathrm{SU}\left(n^{3} / 2\right)$ might be beneficial. We have not been able to make too much use of this, however. Instead simplifications happen when one works with raising/lowering operators that transform in 
$O(n)^{2} \times \mathrm{SU}(n / 2)$. This is perhaps not surprising because we suspect this is the biggest symmetry group of the Clifford raising/lowering operators that can be contained within the symmetry group $O(n)^{3}$ of the theory.

In particular, the Hamiltonian can be written in terms of these redefined tensors as follows:

$$
\begin{aligned}
H= & \sum \psi^{i j k^{+}} \psi^{i l m^{+}} \psi^{n j m^{-}} \psi^{n l k^{-}}+\psi^{i j k^{+}} \psi^{i l m^{-}} \psi^{n j m^{+}} \psi^{n l k^{-}} \\
& +\psi^{i j k^{-}} \psi^{i l m^{+}} \psi^{n j m^{-}} \psi^{n l k^{+}}+\psi^{i j k^{-}} \psi^{i l m^{-}} \psi^{n j m^{+}} \psi^{n l k^{+}}
\end{aligned}
$$

Note that a generic definition of raising/lowering operators will completely scramble the Hamiltonian, so let us take a moment to admire the fact that this form is in fact quite simple. In fact, this can be further simplified using the anti-commutation relations as follows:

$$
H=\frac{n^{4}}{4}+\sum 2\left(\psi^{i j k^{+}} \psi^{i l m^{+}} \psi^{n j m^{-}} \psi^{n l k^{-}}-\psi^{i j k^{+}} \psi^{n j m^{+}} \psi^{i l m^{-}} \psi^{n l k^{-}}\right)
$$

\subsection{Level 0 and level 1}

A Clifford representation is constructed by starting with the lowest weight state |\rangle to be annihilated by all the annihilation operators $\psi^{i j k^{-}}$i.e.,

$$
\psi^{i j k^{-}}|\rangle=0
$$

Note also that this condition immediately implies that the lowest weight state is an eigenstate of the Hamiltonian with the eigenvalue $\frac{n^{4}}{4}$. Thus we have already identified an eigenstate and its eigenvalue.

The rest of the Hilbert space can be generated by acting with the creation operators on |\rangle . So, a general state at level $r$ can be written as:

$$
\psi^{i_{1} j_{1} k_{1}^{+}} \psi^{i_{2} j_{2} k_{2}^{+}} \ldots \psi^{i_{r} j_{r} k_{r}^{+}}|\rangle
$$

Note that there are $\left(\begin{array}{c}n^{3} / 2 \\ r\end{array}\right)$ such states possible. So, the total number of states at all $n^{3} / 2$ levels together is equal to $2^{n^{3} / 2}$.

Now that we have a basis of states, we proceed to identify the eigenstates of the Hamiltonian. Before doing that, it is useful to note the following identity:

$$
\begin{aligned}
\psi^{l m n^{-}} \psi^{i j k^{-}} \psi^{i_{1} j_{1} k_{1}^{+}} \psi^{i_{2} j_{2} k_{2}^{+}} \ldots \psi^{i_{r} j_{r} k_{r}^{+}}|\rangle \\
=\left(\delta^{i i_{1}} \delta^{j j_{1}} \delta^{k k_{1}} \delta^{l i_{2}} \delta^{m j_{2}} \delta^{n k_{2}}-\delta^{i i_{2}} \delta^{j j_{2}} \delta^{k k_{2}} \delta^{l i_{1}} \delta^{m j_{1}} \delta^{n k_{1}}\right) \psi^{i_{3} j_{3} k_{3}^{+}} \psi^{i_{4} j_{4} k_{4}^{+}} \ldots \psi^{i_{r} j_{r} k_{r}^{+}}|\rangle \\
\quad+\left(\delta^{i i_{3}} \delta^{j j_{3}} \delta^{k k_{3}} \delta^{l i_{1}} \delta^{m j_{1}} \delta^{n k_{1}}-\delta^{i i_{1}} \delta^{j j_{1}} \delta^{k k_{1}} \delta^{l i_{3}} \delta^{m j_{3}} \delta^{n k_{3}}\right) \psi^{i_{2} j_{2} k_{2}^{+}} \psi^{i_{4} j_{4} k_{4}^{+}} \ldots \psi^{i_{r} j_{r} k_{r}^{+}}|\rangle \\
\quad+\left(\delta^{i i_{2}} \delta^{j j_{2}} \delta^{k k_{2}} \delta^{l i_{3}} \delta^{m j_{3}} \delta^{n k_{3}}-\delta^{i i_{3}} \delta^{j j_{3}} \delta^{k k_{3}} \delta^{l i_{2}} \delta^{m j_{2}} \delta^{n k_{2}}\right) \psi^{i_{1} j_{1} k_{1}^{+}} \psi^{i_{4} j_{4} k_{4}^{+}} \ldots \psi^{i_{r} j_{r} k_{r}^{+}}|\rangle \\
\quad+\ldots
\end{aligned}
$$

The equation above leads to a crucial fact: namely that the Hamiltonian commutes with the Clifford level. It also immediately demonstrates that the generic level 1 states are also 
annihilated by the Hamiltonian, which means all of them are eigenstates with eigenvalue zero as well.

In what follows, our basic strategy will be to use this fact to try and diagonalize the Hamiltonian level by level.

\subsection{Level 2 and higher}

To get some intuition, we will first identify the eigenstates at level $r=2$. Acting with the Hamiltonian on the state $\psi^{i_{1} j_{1} k_{1}^{+}} \psi^{i_{2} j_{2} k_{2}^{+}}|\rangle$, we obtain the following:

$$
\left(H-\frac{n^{4}}{4}\right)\left(\psi^{i_{1} j_{1} k_{1}^{+}} \psi^{i_{2} j_{2} k_{2}^{+}}|\rangle\right)=4\left[\sum_{i} \psi^{i j_{2} k_{1}^{+}} \psi^{i j_{1} k_{2}^{+}} \delta^{i_{1} i_{2}}-\sum_{j} \psi^{i_{2} j k_{1}^{+}} \psi^{i_{1} j k_{2}^{+}} \delta^{j_{1} j_{2}}\right]|\rangle
$$

The above equation implies that the level 2 states with neither $i_{1}=i_{2}$ nor $j_{1}=j_{2}$ are eigenstates of the Hamiltonian with the same eigenvalue as that of the lowest weight state. This is the beginnings of an observation that we will apply systematically. The basic point is that the Hamiltonian is a singlet, and therefore if one can compare the representation content (under the $O(n)^{2} \times \mathrm{SU}(n / 2)$ symmetry that is manifest) on both sides of the equation, we would have solved the problem of diagonalization.

Level 2 captures the essential structure of the action of the Hamiltonian on a level $r$ state. To see this we present the analogous equation at Clifford levels 3 , for comparison. The action of Hamiltonian on a generic state at level $r=3$ is given by:

$$
\begin{aligned}
\left(H-\frac{n^{4}}{4}\right) & \left(\psi^{i_{1} j_{1} k_{1}^{+}} \psi^{i_{2} j_{2} k_{2}^{+}} \psi^{i_{3} j_{3} k_{3}^{+}}\right)|\rangle \\
= & 4\left[\sum_{i} \psi^{i j_{2} k_{1}^{+}} \psi^{i j_{1} k_{2}^{+}} \delta^{i_{1} i_{2}}-\sum_{j} \psi^{i_{2} j k_{1}^{+}} \psi^{i_{1} j k_{2}^{+}} \delta^{j_{1} j_{2}}\right] \psi^{i_{3} j_{3} k_{3}^{+}}|\rangle \\
& +4\left[\sum_{i} \psi^{i j_{1} k_{3}^{+}} \psi^{i j_{3} k_{1}^{+}} \delta^{i_{3} i_{1}}-\sum_{j} \psi^{i_{1} j k_{3}^{+}} \psi^{i_{3} j k_{1}^{+}} \delta^{j_{3} j_{1}}\right] \psi^{i_{2} j_{2} k_{2}^{+}}|\rangle \\
& +4\left[\sum_{i} \psi^{i j_{3} k_{2}^{+}} \psi^{i j_{2} k_{3}^{+}} \delta^{i_{2} i_{3}}-\sum_{j} \psi^{i_{3} j k_{2}^{+}} \psi^{i_{2} j k_{3}^{+}} \delta^{j_{2} j_{3}}\right] \psi^{i_{1} j_{1} k_{1}^{+}}|\rangle
\end{aligned}
$$

This can easily be generalized to general level. The point about these equations is that they relate representation content on either side. (The Hamiltonian is obviously a singlet under the symmetry of the theory.) So we will write the general equation in a representation theoretic language that makes this point immediate, and show that it is trivial to solve it if we know the representation breakdown. For level 2 this is easy, so we will do it explicitly. For higher and higher levels, the problem becomes more complicated. 


\section{Diagonalization via Young tableaux}

For a general level $m$, the action of the Hamiltonian is given by:

$$
\begin{aligned}
& \left(H-\frac{n^{4}}{4}\right)\left(i_{1} \otimes i_{2} \otimes \ldots \otimes i_{m}, j_{1} \otimes j_{2} \otimes \ldots \otimes j_{m}, k_{1}, k_{2}, \ldots k_{m}\right) \\
& =4 \sum_{p<q}(-1)^{p+q-1}(n)\left[\left(\bullet_{i_{p} i_{q}}, j_{q} \otimes j_{p}, k_{p}, k_{q}\right)-\left(i_{q} \otimes i_{p}, \bullet_{j_{p} j_{q}}, k_{p}, k_{q}\right)\right] \\
& \otimes(\underbrace{i_{1} \otimes i_{2} \otimes \ldots \otimes i_{m}}_{\text {nо } i_{p} \& i_{q}}, \underbrace{j_{1} \otimes j_{2} \otimes \ldots \otimes j_{m}}_{\text {nо } j_{p} \& j_{q}}, \underbrace{k_{1}, k_{2}, \ldots k_{m}}_{\text {no } k_{p} \& k_{q}})
\end{aligned}
$$

We have written the equation now by reverting to the representation theoretic language of Young tableaux. It is straightforward to check that this is correct by directly acting with the Hamiltonian at general level. It is also easy to check that it reduces to the previous expressions at level 2 and 3 (after translating to the previous notation).

To explain the notation, and to demonstrate the general idea, we solve the level 2 case in this language. The indices are written according to $O(n)_{i} \times O(n)_{j} \times \mathrm{SU}(n / 2)_{k}$, where $i, j$ and $k$ refer to the first, second and third indices of the fermionic tensors. Let us start with the equation (2.10) which we present here for convenience:

$$
\left(H-\frac{n^{4}}{4}\right)\left(\psi^{i_{1} j_{1} k_{1}^{+}} \psi^{i_{2} j_{2} k_{2}^{+}}|\rangle\right)=4\left[\sum_{i} \psi^{i j_{2} k_{1}^{+}} \psi^{i j_{1} k_{2}^{+}} \delta^{i_{1} i_{2}}-\sum_{j} \psi^{i_{2} j k_{1}^{+}} \psi^{i_{1} j k_{2}^{+}} \delta^{j_{1} j_{2}}\right]|\rangle
$$

The Hamiltonian is a scalar under $O(n)_{i} \times O(n)_{j} \times \mathrm{SU}(n / 2)_{k}$. So, the 1.h.s. in terms of Young tableaux is given by:

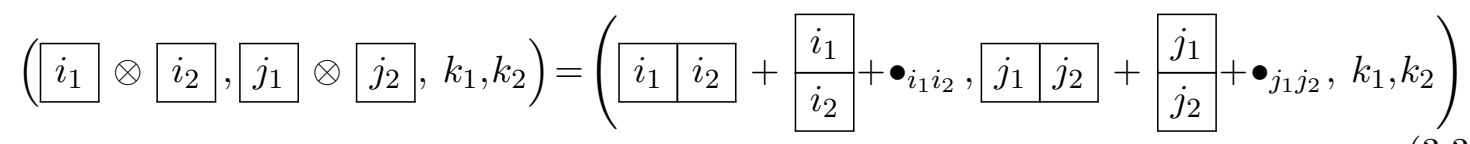

where we have defined

$$
\bullet_{i_{1} i_{2}}=\frac{\delta^{i_{1} i_{2}}}{n} \operatorname{Tr}()^{i i}
$$

As far as the r.h.s. is concerned, only some of the above diagrams show up and are given as:

$$
\left(n \bullet \bullet_{i_{1} i_{2}}, \begin{array}{l|l|}
j_{2} & j_{1} \\
\hline j_{2} \\
\hline j_{1}
\end{array}+n \bullet \bullet_{j_{1} j_{2}}, k_{1}, k_{2}\right)-\left(\begin{array}{l|l}
\hline i_{2} & i_{1} \\
\hline i_{2} \\
\hline i_{1}
\end{array}+n \bullet \bullet_{i_{1} i_{2}}, n \bullet_{j_{1} j_{2}}, k_{1}, k_{2}\right)
$$

Upon comparing the l.h.s. and r.h.s., we can conclude that the following states have an eigenvalue of " $+4 n$ ":

$$
\begin{aligned}
& \left(H-\frac{n^{4}}{4}\right)\left(\bullet_{i_{1} i_{2}}, \begin{array}{l|l}
j_{1} & j_{2}
\end{array}, k_{1}, k_{2}\right)=+4 n\left(\bullet_{i_{1} i_{2}}, \begin{array}{|l|l|}
j_{1} & j_{2} \\
\hline
\end{array}, k_{1}, k_{2}\right) \\
& \left(H-\frac{n^{4}}{4}\right)\left(\frac{i_{1}}{i_{2}}, \bullet_{j_{1} j_{2}}, k_{1}, k_{2}\right)=+4 n\left(\frac{i_{1}}{i_{2}}, \bullet_{j_{1} j_{2}}, k_{1}, k_{2}\right)
\end{aligned}
$$


Further, the following two sets of states have an eigenvalue of " $-4 n$ ":

$$
\begin{gathered}
\left(H-\frac{n^{4}}{4}\right)\left(\bullet_{i_{1} i_{2}}, \frac{j_{1}}{j_{2}}, k_{1}, k_{2}\right)=-4 n\left(\bullet \bullet_{i_{1} i_{2}}, \frac{j_{1}}{j_{2}}, k_{1}, k_{2}\right) \\
\left(H-\frac{n^{4}}{4}\right)\left(\begin{array}{ll}
i_{1} & i_{2}
\end{array}, \bullet_{j_{1} j_{2}}, k_{1}, k_{2}\right)=-4 n\left(\begin{array}{ll}
i_{1} & \left.i_{2}, \bullet_{j_{1} j_{2}}, k_{1}, k_{2}\right)
\end{array}\right.
\end{gathered}
$$

The rest of the states have a zero eigenvalue. These results can also be re-done directly with more indices etc., and we show that in appendix A for convenience in understanding our notation.

The idea then is to compare the representation content on either side of (3.1) and that will yield the eigenstates directly. Note that the eigenvalues and the eigenfunctions are all purely determined by representation theory at this stage.

\subsection{Comments}

We note a few relevant points here, and leave a detailed study of the higher levels for future work.

What we have seen is that the diagonalization of the Hamiltonian reduces to a group theory problem, where we are instructed to match representations in (3.1) level by level. The basic ingredients for combining representations in the Young tableaux language are the Littlewood-Richardson rules. Here we need them for the $O(n)^{2} \times \mathrm{SU}(n / 2)$ group. The $\mathrm{SU}(n / 2)$ representation structure of equation (3.1) is trivial (modulo one caveat we will discuss), so the question is really about combining $O(n) \times O(n)$ representations. The Littlewood-Richardson rules in the $O(n)$ case are known ${ }^{2}$ [86] and so this is in principle straightforward. What we have done in level 2 is an example of such an operation. The trouble of course is that the representations grow as we go to higher and higher levels (even though they will turn around by level $n^{3} / 4$ because of the finiteness of the Clifford representation).

This is straightforward enough, but there are two things one needs to be careful about. One is that the tensors we are combining here are fermions, so they should be treated as anti-commuting objects. This becomes important when the tensors that are being combined are to be treated as identical, which happens when the value of the $k_{i}$ indices on two of the tensors that are being combined happen to be the same. When that happens, some of the representations that result are identically zero. We illustrate this point for level 2, by explicitly showing the count of the number of states that fall into each representation in an appendix.

The details of the "plethysms" that we will need will be discussed in the forthcoming paper [85].

\footnotetext{
${ }^{2}$ More precisely, $\mathrm{SO}(n)$ case. But this difference only affects the details of the calculations, not the actual result.
} 


\section{Conclusion}

Our explicit example here has been for the specific case of uncolored tensor models with a symmetry group of even rank. But the crucial ingredient in our strategy was more general: we exploited the interplay between the level structure of the spinor representation and the Hamiltonian. We found that the two commute, which simplified the problem enormously. But this may not be necessary for solvability - if the algebra of the Hamiltonian and the level have a suitable structure, there could be a systematic way to exploit it. It will be interesting to see if other holographic tensor models (in particular, the odd rank version of the theory considered here) lead to solvability in some such approach. Also, it seems highly plausible that a strategy like the one presented here might go through for the colored tensor models as well [85].

Another comment worth making is that we have not used the full symmetries of the Hamiltonian. In particular, the full $O(n)^{3}$ symmetry of the Hamiltonian is not manifest in our Clifford representation approach. This raises two points.

- One should be able to check that the full spectrum that one obtains at the end has the full symmetry. (Note that the level construction is what breaks manifest symmetry, so one will not see the full symmetry realized in any one level alone.)

- Is there a way to set up the problem so that one can exploit the symmetries more systematically than we have?

One sinister way in which this program can fail (at least for the case of the even $n$ tensor models we consider here) is if the theory for even $n$ and odd $n$ are qualitatively different, and only the odd $n$ theory behaves like SYK in the large $n$ limit. The explicit case diagonalized in [31] and found to have some chaos-like behavior was an odd $n$ case, the higher even $n$ cases were inaccessible to numerical diaogonalization. We find the scenario that the odd and even cases are qualitatively different to be quite unlikely (note also that in the colored model, the even case already exhibited chaos [30]), afterall it seems reasonable to expect that the large$N$ limit of the even and odd cases should match. But we are not sure if this is a watertight way to rule out this malicious possibility, which is why we have decided to menion it here.

There is another well-known context [81-84] in holography, where Young tableaux (and very closely related Schur polynomials) have emerged as a fundamental ingredient. This is in the reconstruction of BPS states/geometries in the bulk, via the gauge singlets in the boundary. Note that the Young tableaux that emerge in our construction are different. Roughly speaking, the construction of [81-84] present the "closed string" perspetive on the problem, while ours is an "open string" picture. Of course, once one follows through the program we have outlined here to completion, it will be very interesting to identify the gauge singlet states and operators and to explicitly reconstruct the bulk. It seems highly plausible that such a construction will clarify the relationship between the two distinct avatars of Young tableaux in these theories.

We conclude with one further comment. In holographic theories, one is only interested in gauge singlets on the boundary. One way to implement this is to note that after diagonalization, one only keeps the gauge singlet states. But one can also reverse the order of 
this process and look only for eigenstates that are gauge singlet states to start with. From that perspective, the problem we have tackled here is harder than it needs to be. We will discuss this issue further in [85].

There are clearly numerous questions that one can address with a full solution of a holographic quantum theory. We will exercise restraint in taking stock of such a wishlist here.

\section{Acknowledgments}

CK thanks Justin Raj David and Ramalingam Loganayagam for discussions. We thank P. N. Bala Subramanian for a closely related collaboration [85].

\section{A Explicit diagonalization of level 2}

In the main body of the paper, we presented the eigenstates and eignevalues at level 2 working directly with Young tableaux. In this appendix, we reinterpret the same calculation in terms of explicit creation/annihilation operators etc. at level 2.

A general state at level 2 is of the form $\psi^{i_{1} j_{1} k_{1}} \psi^{i_{2} j_{2} k_{2}}$. Hamiltonian acting on such a state gives us:

$$
\left(H-\frac{n^{4}}{4}\right)\left(\psi^{i_{1} j_{1} k_{1}^{+}} \psi^{i_{2} j_{2} k_{2}^{+}}\right)|\rangle=4\left[\sum_{i} \psi^{i j_{2} k_{1}^{+}} \psi^{i j_{1} k_{2}^{+}} \delta^{i_{1} i_{2}}-\sum_{j} \psi^{i_{2} j k_{1}^{+}} \psi^{i_{1} j k_{2}^{+}} \delta^{j_{1} j_{2}}\right]|\rangle
$$

We now take a trace over the indices $i_{1}$ and $i_{2}$ i.e., we multiply the above equation by $\delta^{i_{1} i_{2}}$ and then take a sum over $i_{1}$ and $i_{2}$. Doing so, we will get:

$$
\left(H-\frac{n^{4}}{4}\right) \sum_{i}\left(\psi^{i j_{1} k_{1}^{+}} \psi^{i j_{2} k_{2}^{+}}\right)|\rangle=\left[4 n \sum_{i} \psi^{i j_{2} k_{1}^{+}} \psi^{i j_{1} k_{2}^{+}}-4 \sum_{j, i} \psi^{i j k_{1}^{+}} \psi^{i j k_{2}^{+}} \delta^{j_{1} j_{2}}\right]|\rangle
$$

Similarly, a trace over the indices $j_{1}$ and $j_{2}$ gives us:

$$
\left(H-\frac{n^{4}}{4}\right) \sum_{j}\left(\psi^{i_{1} j k_{1}^{+}} \psi^{i_{2} j k_{2}^{+}}\right)|\rangle=\left[4 \sum_{i, j} \psi^{i j k_{1}^{+}} \psi^{i j k_{2}^{+}} \delta^{i_{1} i_{2}}-4 n \sum_{j} \psi^{i_{2} j k_{1}^{+}} \psi^{i_{1} j k_{2}^{+}}\right]|\rangle
$$

Taking a linear combination of the above three equations, we get:

$$
\left(H-\frac{n^{4}}{4}\right)\left[\psi^{i_{1} j_{1} k_{1}^{+}} \psi^{i_{2} j_{2} k_{2}^{+}}-\frac{1}{n}\left(\sum_{i} \psi^{i j_{1} k_{1}^{+}} \psi^{i j_{2} k_{2}^{+}} \delta^{i_{1} i_{2}}+\sum_{j} \psi^{i_{1} j k_{1}^{+}} \psi^{i_{2} j k_{2}^{+}} \delta^{j_{1} j_{2}}\right)\right]|\rangle=0
$$

So, the traceless part of a generic second level state is an eigenstate of the Hamiltonian with an eigenvalue $\frac{n^{4}}{4}$. 
Further, it can also be shown that

$$
\begin{aligned}
& \left(H-\frac{n^{4}}{4}\right) \sum_{i}\left(\psi^{i j_{1} k_{1}^{+}} \psi^{i j_{2} k_{2}^{+}}-\psi^{i j_{2} k_{1}^{+}} \psi^{i j_{1} k_{2}^{+}}\right)|\rangle=-4 n \sum_{i}\left(\psi^{i j_{1} k_{1}^{+}} \psi^{i j_{2} k_{2}^{+}}-\psi^{i j_{2} k_{1}^{+}} \psi^{i j_{1} k_{2}^{+}}\right)|\rangle \\
& \left(H-\frac{n^{4}}{4}\right) \sum_{j}\left(\psi^{i_{1} j k_{1}^{+}} \psi^{i_{2} j k_{2}^{+}}-\psi^{i_{2} j k_{1}^{+}} \psi^{i_{1} j k_{2}^{+}}\right)|\rangle=+4 n \sum_{j}\left(\psi^{i_{1} j k_{1}^{+}} \psi^{i_{2} j k_{2}^{+}}-\psi^{i_{2} j k_{1}^{+}} \psi^{i_{1} j k_{2}^{+}}\right)|\rangle
\end{aligned}
$$

Taking a trace over both the $i$ 's and $j$ 's, we get an eigenstate with an eigenvalue $\frac{n^{4}}{4}$ :

$$
\left(H-\frac{n^{4}}{4}\right) \sum_{i, j} \psi^{i j k_{1}^{+}} \psi^{i j k_{2}^{+}}|\rangle=0
$$

\section{B Counting at level 2}

In this appendix, we explicitly count the number of states that fall into various irreducible representations at level 2. A generic state at this level is given by:

$$
\left(i_{1} \otimes i_{2}, j_{1} \otimes j_{2}, k_{1}, k_{2}\right)
$$

Note that the total number of states at this level are $\left(\begin{array}{c}n^{3} / 2 \\ 2\end{array}\right)$ as there are $\frac{n^{3}}{2}$ creation operators.

We consider the case of $k_{1} \neq k_{2}$. In this case all the possible irreps show up. So, the possible states are:

$$
\underbrace{\left(\begin{array}{ll}
i_{1} & i_{2} \\
\hline
\end{array}+\begin{array}{l}
i_{1} \\
\hline i_{2}
\end{array}+\bullet_{i_{1} i_{2}}, \begin{array}{l|l}
j_{1} & j_{2} \\
\hline j_{2}
\end{array}+\bullet_{j_{1} j_{2}}, k_{1}, k_{2}\right)}_{n^{2} \times n^{2} \times\left(\begin{array}{c}
n / 2 \\
2
\end{array}\right)}
$$

Since all the irreps corresponding to $i$ 's and $j$ 's are present and $k_{1} \neq k_{2}$, we find that the total number of states with $k_{1} \neq k_{2}$ are $\frac{n^{5}}{8}(n-2)$.

The case of $k_{1}=k_{2}$ is somewhat tricky. Thanks to the antisymmetric nature of the $\psi$ 's, some of the representations are trivially zero. The non trivial states with $k_{1}=k_{2}$ are given by:

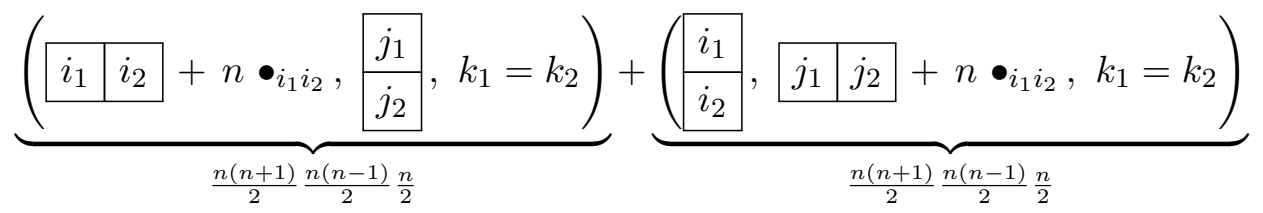

Since the symmetric traceless representation has the dimension of $\frac{n(n+1)}{2}-1$ and the antisymmetric part has the dimension of $\frac{n(n-1)}{2}$, we can see that the states with $k_{1}=k_{2}$ are $\frac{n^{3}}{4}\left(n^{2}-1\right)$ in number. Adding the contributions from both the cases gives the desired value of $\left(\begin{array}{c}n^{3} / 2 \\ 2\end{array}\right)$. 
Open Access. This article is distributed under the terms of the Creative Commons Attribution License (CC-BY 4.0), which permits any use, distribution and reproduction in any medium, provided the original author(s) and source are credited.

\section{References}

[1] R. Gopakumar, What is the simplest gauge-string duality?, arXiv:1104.2386 [INSPIRE].

[2] S. El-Showk and K. Papadodimas, Emergent spacetime and holographic CFTs, JHEP 10 (2012) 106 [arXiv:1101.4163] [INSPIRE].

[3] P.H. Ginsparg and G.W. Moore, Lectures on $2 D$ gravity and $2 D$ string theory, published in YCTP-P23-92, Yale Univ., New Haven CT U.S.A., (1992) and in LA-UR-92-3479 Los Alamos Nat. Lab., Los Alamos NM U.S.A., (1992) [hep-th/9304011] [INSPIRE].

[4] J. McGreevy and H.L. Verlinde, Strings from tachyons: the $c=1$ matrix reloaded, JHEP 12 (2003) 054 [hep-th/0304224] [INSPIRE].

[5] S. Mukhi, Topological matrix models, Liouville matrix model and $c=1$ string theory, hep-th/0310287 [INSPIRE].

[6] M. Kontsevich, Intersection theory on the moduli space of curves and the matrix Airy function, Commun. Math. Phys. 147 (1992) 1 [INSPIRE].

[7] E. Witten, On the structure of the topological phase of two-dimensional gravity, Nucl. Phys. B 340 (1990) 281 [inSPIRE].

[8] J. Distler, 2D quantum gravity, topological field theory and the multicritical matrix models, Nucl. Phys. B 342 (1990) 523 [inSPIRE].

[9] E.P. Verlinde and H.L. Verlinde, A solution of two-dimensional topological quantum gravity, Nucl. Phys. B 348 (1991) 457 [InSPIRE].

[10] D. Gaiotto and L. Rastelli, A paradigm of open/closed duality: Liouville D-branes and the Kontsevich model, JHEP 07 (2005) 053 [hep-th/0312196] [INSPIRE].

[11] R. Gopakumar and C. Vafa, On the gauge theory/geometry correspondence, Adv. Theor. Math. Phys. 3 (1999) 1415 [hep-th/9811131] [INSPIRE].

[12] J.M. Maldacena, Eternal black holes in anti-de Sitter, JHEP 04 (2003) 021 [hep-th/0106112] [INSPIRE].

[13] C. Krishnan, Quantum field theory, black holes and holography, arXiv:1011.5875 [INSPIRE].

[14] J.L.F. Barbon and E. Rabinovici, Very long time scales and black hole thermal equilibrium, JHEP 11 (2003) 047 [hep-th/0308063] [INSPIRE].

[15] T. Anous, T. Hartman, A. Rovai and J. Sonner, Black hole collapse in the 1/c expansion, JHEP 07 (2016) 123 [arXiv: 1603.04856] [INSPIRE].

[16] A.L. Fitzpatrick, J. Kaplan, D. Li and J. Wang, On information loss in $A d S_{3} / C F T_{2}$, JHEP 05 (2016) 109 [arXiv: 1603.08925] [INSPIRE].

[17] E. Berkowitz, E. Rinaldi, M. Hanada, G. Ishiki, S. Shimasaki and P. Vranas, Precision lattice test of the gauge/gravity duality at large- $N$, Phys. Rev. D 94 (2016) 094501 [arXiv: 1606.04951] [INSPIRE].

[18] M. Hanada, Y. Hyakutake, G. Ishiki and J. Nishimura, Holographic description of quantum black hole on a computer, Science 344 (2014) 882 [arXiv:1311.5607] [INSPIRE]. 
[19] V. Pestun et al., Localization techniques in quantum field theories, arXiv:1608.02952 [INSPIRE].

[20] A. Almheiri and J. Polchinski, Models of AdS $S_{2}$ backreaction and holography, JHEP 11 (2015) 014 [arXiv:1402.6334] [INSPIRE].

[21] J. Maldacena and D. Stanford, Remarks on the Sachdev-Ye-Kitaev model, Phys. Rev. D 94 (2016) 106002 [arXiv: 1604.07818] [INSPIRE].

[22] J. Polchinski and V. Rosenhaus, The spectrum in the Sachdev-Ye-Kitaev model, JHEP 04 (2016) 001 [arXiv:1601.06768] [INSPIRE].

[23] J. Maldacena, S.H. Shenker and D. Stanford, A bound on chaos, JHEP 08 (2016) 106 [arXiv: 1503.01409] [INSPIRE].

[24] J.L. Karczmarek, J.M. Maldacena and A. Strominger, Black hole non-formation in the matrix model, JHEP 01 (2006) 039 [hep-th/0411174] [INSPIRE].

[25] B. Michel, J. Polchinski, V. Rosenhaus and S.J. Suh, Four-point function in the IOP matrix model, JHEP 05 (2016) 048 [arXiv: 1602.06422] [INSPIRE].

[26] E. Witten, An SYK-like model without disorder, arXiv:1610.09758 [INSPIRE].

[27] I.R. Klebanov and G. Tarnopolsky, Uncolored random tensors, melon diagrams and the Sachdev-Ye-Kitaev models, Phys. Rev. D 95 (2017) 046004 [arXiv:1611.08915] [InSPIRE].

[28] S. Carrozza and A. Tanasa, $O(N)$ random tensor models, Lett. Math. Phys. 106 (2016) 1531 [arXiv: 1512.06718] [INSPIRE].

[29] V. Bonzom, R. Gurau and V. Rivasseau, Random tensor models in the large- $N$ limit: uncoloring the colored tensor models, Phys. Rev. D 85 (2012) 084037 [arXiv:1202.3637] [INSPIRE].

[30] C. Krishnan, S. Sanyal and P.N. Bala Subramanian, Quantum chaos and holographic tensor models, JHEP 03 (2017) 056 [arXiv: 1612.06330] [INSPIRE].

[31] C. Krishnan, K.V.P. Kumar and S. Sanyal, Random matrices and holographic tensor models, JHEP 06 (2017) 036 [arXiv:1703.08155] [INSPIRE].

[32] R. Gurau, The complete $1 / N$ expansion of a $S Y K$-like tensor model, Nucl. Phys. B 916 (2017) 386 [arXiv:1611.04032] [INSPIRE].

[33] R. Gurau, Quenched equals annealed at leading order in the colored SYK model, arXiv: 1702.04228 [INSPIRE].

[34] S. Chaudhuri, V.I. Giraldo-Rivera, A. Joseph, R. Loganayagam and J. Yoon, Abelian tensor models on the lattice, arXiv:1705.01930 [INSPIRE].

[35] P. Narayan and J. Yoon, SYK-like tensor models on the lattice, JHEP 08 (2017) 083 [arXiv: 1705.01554] [INSPIRE].

[36] Y. Liu, M.A. Nowak and I. Zahed, Disorder in the Sachdev-Yee-Kitaev model, Phys. Lett. B 773 (2017) 647 [arXiv: 1612.05233] [INSPIRE].

[37] C. Peng, M. Spradlin and A. Volovich, A supersymmetric SYK-like tensor model, JHEP 05 (2017) 062 [arXiv: 1612.03851] [INSPIRE].

[38] R.A. Davison, W. Fu, A. Georges, Y. Gu, K. Jensen and S. Sachdev, Thermoelectric transport in disordered metals without quasiparticles: the Sachdev-Ye-Kitaev models and holography, Phys. Rev. B 95 (2017) 155131 [arXiv:1612.00849] [INSPIRE]. 
[39] T. Nishinaka and S. Terashima, A note on Sachdev-Ye-Kitaev like model without random coupling, arXiv:1611.10290 [INSPIRE].

[40] W. Fu, D. Gaiotto, J. Maldacena and S. Sachdev, Supersymmetric Sachdev-Ye-Kitaev models, Phys. Rev. D 95 (2017) 026009 [Addendum ibid. D 95 (2017) 069904] [arXiv: 1610.08917] [INSPIRE].

[41] S. Banerjee and E. Altman, Solvable model for a dynamical quantum phase transition from fast to slow scrambling, Phys. Rev. B 95 (2017) 134302 [arXiv:1610.04619] [INSPIRE].

[42] M. Berkooz, P. Narayan, M. Rozali and J. Simón, Higher dimensional generalizations of the SYK model, JHEP 01 (2017) 138 [arXiv:1610.02422] [INSPIRE].

[43] D.J. Gross and V. Rosenhaus, A generalization of Sachdev-Ye-Kitaev, JHEP 02 (2017) 093 [arXiv: 1610.01569$]$ [INSPIRE].

[44] Y. Gu, X.-L. Qi and D. Stanford, Local criticality, diffusion and chaos in generalized Sachdev-Ye-Kitaev models, JHEP 05 (2017) 125 [arXiv: 1609. 07832] [INSPIRE].

[45] A. Jevicki and K. Suzuki, Bi-local holography in the SYK model: perturbations, JHEP 11 (2016) 046 [arXiv: 1608.07567] [INSPIRE].

[46] A. Jevicki, K. Suzuki and J. Yoon, Bi-local holography in the SYK model, JHEP 07 (2016) 007 [arXiv: 1603.06246] [INSPIRE].

[47] A. Almheiri and J. Polchinski, Models of AdS $S_{2}$ backreaction and holography, JHEP 11 (2015) 014 [arXiv: 1402.6334] [INSPIRE].

[48] J. Maldacena, D. Stanford and Z. Yang, Conformal symmetry and its breaking in two dimensional nearly anti-de-Sitter space, Prog. Theor. Exp. Phys. 2016 (2016) 12C104 [arXiv: 1606.01857] [INSPIRE].

[49] M. Cvetič and I. Papadimitriou, AdS $S_{2}$ holographic dictionary, JHEP 12 (2016) 008 [Erratum ibid. 01 (2017) 120] [arXiv:1608.07018] [INSPIRE].

[50] J.M. Magan, Decoherence and microscopic diffusion at SYK, arXiv:1612.06765 [INSPIRE].

[51] F. Ferrari, The large D limit of planar diagrams, arXiv:1701.01171 [INSPIRE].

[52] A.M. García-García and J.J.M. Verbaarschot, Analytical spectral density of the Sachdev-Ye-Kitaev model at finite N, Phys. Rev. D 96 (2017) 066012 [arXiv:1701.06593] [INSPIRE].

[53] T. Anous and C. Cogburn, Mini-BFSS in Silico, arXiv:1701.07511 [INSPIRE].

[54] W.W. Ho and D. Radicevic, The ergodicity landscape of quantum theories, arXiv: 1701.08777 [INSPIRE].

[55] T. Li, J. Liu, Y. Xin and Y. Zhou, Supersymmetric SYK model and random matrix theory, JHEP 06 (2017) 111 [arXiv:1702.01738] [INSPIRE].

[56] R. Gurau, Quenched equals annealed at leading order in the colored SYK model, arXiv: 1702.04228 [INSPIRE].

[57] M. Berkooz, P. Narayan, M. Rozali and J. Simón, Comments on the random Thirring model, JHEP 09 (2017) 057 [arXiv: 1702.05105] [INSPIRE].

[58] V. Bonzom, L. Lionni and A. Tanasa, Diagrammatics of a colored SYK model and of an SYK-like tensor model, leading and next-to-leading orders, J. Math. Phys. 58 (2017) 052301 [arXiv: 1702.06944] [INSPIRE]. 
[59] D.J. Gross and V. Rosenhaus, The bulk dual of SYK: cubic couplings, JHEP 05 (2017) 092 [arXiv: 1702.08016] [INSPIRE].

[60] V. Rivasseau, Loop vertex expansion for higher order interactions, arXiv:1702.07602 [INSPIRE].

[61] S.-K. Jian and H. Yao, Solvable SYK models in higher dimensions: a new type of many-body localization transition, arXiv:1703.02051 [INSPIRE].

[62] H. Itoyama, A. Mironov and A. Morozov, Rainbow tensor model with enhanced symmetry and extreme melonic dominance, Phys. Lett. B 771 (2017) 180 [arXiv:1703.04983] [INSPIRE].

[63] V. Rivasseau and F. Vignes-Tourneret, Constructive tensor field theory: the $T_{4}^{4}$ model, arXiv:1703.06510 [INSPIRE].

[64] K. Jensen, Chaos in AdS 2 holography, Phys. Rev. Lett. 117 (2016) 111601 [arXiv: 1605. 06098] [INSPIRE].

[65] D. Stanford and E. Witten, Fermionic localization of the Schwarzian theory, JHEP 10 (2017) 008 [arXiv: 1703.04612] [INSPIRE].

[66] G. Mandal, P. Nayak and S.R. Wadia, Coadjoint orbit action of Virasoro group and two-dimensional quantum gravity dual to SYK/tensor models, arXiv:1702.04266 [INSPIRE].

[67] S. Förste and I. Golla, Nearly AdS 2 SUGRA and the super-Schwarzian, Phys. Lett. B 771 (2017) 157 [arXiv:1703.10969] [INSPIRE].

[68] C. Peng, Vector models and generalized SYK models, JHEP 05 (2017) 129 [arXiv: 1704.04223] [INSPIRE].

[69] H. Itoyama, A. Mironov and A. Morozov, Ward identities and combinatorics of rainbow tensor models, JHEP 06 (2017) 115 [arXiv: 1704.08648] [INSPIRE].

[70] X. Chen, R. Fan, Y. Chen, H. Zhai and P. Zhang, Competition between chaotic and non-chaotic phases in a quadratically coupled Sachdev-Ye-Kitaev model, arXiv:1705.03406 [INSPIRE].

[71] Y. Chen, H. Zhai and P. Zhang, Tunable quantum chaos in the Sachdev-Ye-Kitaev model coupled to a thermal bath, JHEP 07 (2017) 150 [arXiv: 1705.09818] [INSPIRE].

[72] S. Dartois, H. Erbin and S. Mondal, Conformality of $1 / N$ corrections in SYK-like models, arXiv:1706.00412 [INSPIRE].

[73] T. Kanazawa and T. Wettig, Complete random matrix classification of SYK models with $N=0,1$ and 2 supersymmetry, JHEP 09 (2017) 050 [arXiv:1706.03044] [INSPIRE].

[74] A. Mironov and A. Morozov, Correlators in tensor models from character calculus, Phys. Lett. B 774 (2017) 210 [arXiv:1706.03667] [INSPIRE].

[75] S.R. Das, A. Jevicki and K. Suzuki, Three dimensional view of the SYK/AdS duality, JHEP 09 (2017) 017 [arXiv: 1704.07208] [INSPIRE].

[76] R. Gurau, Invitation to random tensors, SIGMA 12 (2016) 094 [arXiv:1609.06439] [INSPIRE].

[77] V. Bonzom, R. Gurau, A. Riello and V. Rivasseau, Critical behavior of colored tensor models in the large-N limit, Nucl. Phys. B 853 (2011) 174 [arXiv:1105.3122] [INSPIRE].

[78] R. Gurau, The complete $1 / N$ expansion of colored tensor models in arbitrary dimension, Annales Henri Poincaré 13 (2012) 399 [arXiv:1102.5759] [INSPIRE]. 
[79] R. Gurau and V. Rivasseau, The $1 / N$ expansion of colored tensor models in arbitrary dimension, EPL 95 (2011) 50004 [arXiv:1101.4182] [INSPIRE].

[80] R. Gurau, The $1 / N$ expansion of colored tensor models, Annales Henri Poincaré 12 (2011) 829 [arXiv: 1011.2726] [INSPIRE].

[81] H. Lin, O. Lunin and J.M. Maldacena, Bubbling AdS space and 1/2 BPS geometries, JHEP 10 (2004) 025 [hep-th/0409174] [INSPIRE].

[82] D. Berenstein, A toy model for the AdS/CFT correspondence, JHEP 07 (2004) 018 [hep-th/0403110] [INSPIRE].

[83] S. Corley, A. Jevicki and S. Ramgoolam, Exact correlators of giant gravitons from dual $N=4$ SYM theory, Adv. Theor. Math. Phys. 5 (2002) 809 [hep-th/0111222] [INSPIRE].

[84] R. de Mello Koch, Geometries from Young diagrams, JHEP 11 (2008) 061 [arXiv:0806.0685] [INSPIRE].

[85] C. Krishnan and K.V. Pavan Kumar, An exact tensor model hologram, to appear.

[86] V.E. Didenko and E.D. Skvortsov, Elements of Vasiliev theory, arXiv:1401.2975 [INSPIRE]. 